\title{
Interactive comment on "A Global Compilation of U-series Dated Fossil Coral Sea-level Indicators for the Last Interglacial Period (MIS 5e)" by Peter M. Chutcharavan and Andrea Dutton
}

Anonymous Referee \#2

Received and published: 20 March 2021

\section{General comments}

The manuscript is well written and clearly structured. It presents helpful geochemical screening protocols of Last Interglacial (LIG; MIS 5e) corals, and therefore provides an important basis for the present MIS 5 e sea-level database.

I am concerned about the overlap of the present manuscript with other manuscripts of the WALIS Special Issue addressing LIG corals as sea-level indicators in various geographic regions. The primary focus of the manuscript by Chutcharavan and Dutton is on the U-series aspect of LIG corals, but in Section 2.4 the authors also focus on growth position and paleowater depth interpretations as well as tectonics and Glacial 
Isostatic Adjustment (GIA). The discussion on paleowater depth in Section 2.4 appears to be beyond the scope of the paper as other manuscripts of this Special Issue should address this topic so that it should not be part of this manuscript. The authors should have exclusively focused on the geochemical screening to avoid any overlap with other manuscripts of the WALIS Special Issue. In order to avoid redundancy and therefore not to confuse the audience, the authors should refer to the other WALIS databases and manuscripts addressing uplift/subsidence rates, paleowater depths and growth position (in situ vs reworked samples) of LIG corals in the respective geographic regions.

As the manuscript claims to be a global compilation of LIG sea-level indicators, the authors should explore if they did the geochemical screening for all datapoints summarized in the other articles of the WALIS Special Issue, e.g., the Western Mediterranean, Madagascar, Belize and some islands/archipelagos in the tropical Pacific Ocean.

I therefore recommend that this paper could be accepted after moderate revisions regarding the aspects detailed above and the specific comments that I listed below.

\section{Specific comments}

- The authors should not cite Hibbert et al. (2016) for the modern depth distribution of coral taxa, but refer to the Ocean Biogeographical Information System (OBIS) as Hibbert et al. (2016) use information from the OBIS. See lines 285, 299, 472, $515,548,637,652,683,705$ and 772.

- Lines 258-259: "Growth position is usually interpreted as expressing greater confidence than in situ, as it implies that the coral is in the correct growth orientation or that a clear basal attachment to the reef substrate is visible at the outcrop scale." => This introduces some uncertainty as some corals in growth position 
might not be in situ; it is not unlikely that corals have been transported, but still look like being in growth position. I agree that there is a problem with inconsistent terminology (in situ and growth position) in the literature and in addition many original studies do not mention if the dated corals have been collected in growth position/in situ.

- Lines 395-396: how many of the samples passing the two screening protocols are in situ?

- Lines 570-574: Thomas et al. (2009) should not be included in the WALIS database as the dataset (13 datapoints) does not present MIS $5 \mathrm{e}$.

- Lines 618-619: do you consider only mass spectrometry? If not, there are many earlier studies who dated the Waimanalo formation.

- Line 662: Sample KE12-001 is not a microatoll. According to Kennedy et al. (2012), the Last Interglacial "sample was from a 2 m-high coral head and was associated with gravel clasts of other massive corals $>0.5 \mathrm{~m}$ in diameter".

- Lines 666-667: Probably you only consider studies providing mass spectrometry U-series ages. If so, please specify. If not, Omura et al. (1994) should be mentioned as they also dated the reef terraces on Huon Peninsula (alpha spectrometry).

\section{Technical corrections}

1) General formatting issues:

Be consistent with text justified or left-aligned.

Be consistent with capitalizing words in the headings.

Be consistent with writing numbers $\leq 10$, e.g., lines 437, 499, 519, 525, 526, 529, 535, 


\section{2) Further formatting issues:}

Line 17: add a comma before "2020"

Lines 26-27: merge references

Edwards et al., 1987a; Edwards et al., 1987b => Edwards et al., 1987a, b

Interactive

comment

Stirling et al., 1995; Stirling et al., 1998 => Stirling et al., 1995, 1998

Line 63: add a comma before "2020"

Lines 74-76: Correct the formatting of the list, i.e., do not capitalize the first letter of the items (2) and (3); separate the items by commas or semicolons and finish item (3) with a full stop.

Line 93: add hyphen to "sea-level history"

Line 123: "in situ" in italics

Line 172: delete "that" as it appears twice

Lines 187 and 204: Do not capitalize "detrital" as it is not at the beginning of a new sentence.

Line 203: Do not capitalize "calcite" as it is not at the beginning of a new sentence.

Line 258: full stop after quotation mark

Figure 5 caption: genera in italics

Line 324: add "to" after "comparison"

Section 2.5.3: Do not use the abbreviation "DT" without explaining it once at the beginning of this section (could be done in line 370).

Lines 385-392: Use semicolons to separate the listed items and finish item (7) with a

full stop. Do not capitalize the first letter of the items.

Line 437: analyses "in" total

Line 444: comma after "In total"

Line 467: corals "in" total

Line 505: correct writing of Curaçao

Line 507: comma after "In total" 
Line 561 and 574: Thomas et al., 2009; a or b?

Line 757: O'Leary et al., 2008b, 2008a => O'Leary et al., 2008a, b

Line 796: Andersen et al., 2010b, $2008=>$ change the order of the years

Line 803: there is no item $4.3=>$ correct numbering

Line 805: delete space before "scale"

Line 808: "has advanced" => "have advanced"

Interactive

comment

Lines 819: add full stop

\section{3) Mistakes in the database:}

- WALIS U-series ID 1292: ST93-009-001 should be Stein et al. (1993) and not Cutler et al. (2003)

- WALIS U-series ID 1313: ST93-007-003 should be Stein et al. (1993) and not Esat et al. (1999)

- WALIS U-series ID 1664: according to the sample ID OL13 the reference should be O'Leary et al. (2013) instead of Stirling et al. (2001)

- WALIS U-series ID 1763, 1764 and 1765: according to the sample ID SC78 the reference should not be Muhs et al. (2012)

- WALIS U-series ID 2086, 2087 and 2088: according to the sample ID ED87-008 the reference should be Edwards et. (1987) instead of Gallup et al. (1994)

- WALIS U-series ID 2137: according to the sample ID HA91 the reference should be Hamelin et al. (1991) instead of Thompson et al. (2003)

- WALIS U-series ID 2168: change Edwards et al. (1997) to Edwards et al. (1987) 
- WALIS U-series ID 2169, 2170, 2171 and 2172: according to the sample ID GA94 the reference should be Gallup et al. (1994) instead of Edwards et al. (1997)

- WALIS U-series ID 2364: according to the sample ID SC78-004 or analysis ID SZ78-004-002 (which one is correct?) the reference should not be Muhs et al. (2012)

Interactive comment on Earth Syst. Sci. Data Discuss., https://doi.org/10.5194/essd2020-381, 2020. 